\title{
Estimation of Hypervelocity Impact Parameters from Measurements of Optical Flash
}

\author{
A. Goel ${ }^{\mathrm{a}}$, N. Lee ${ }^{\dagger a}$, S. Close ${ }^{\mathrm{a}}$ \\ ${ }^{a}$ Dept. of Aeronautics and Astronautics, Stanford University, Stanford, CA 94305, USA
}

\begin{abstract}
When meteoroids and orbital debris hit satellites or surfaces of other objects in space, an optical flash is generated. Measuring properties of this impact flash can reveal several important properties of the impact phenomenon. In this paper, we present results from hypervelocity impact tests that were carried out at the Max Planck Institute for Nuclear Physics in Heidelberg, Germany. Spherical iron projectiles were shot at targets comprising tungsten, copper, spacecraft solar cells, solar panel substrate and optical solar reflectors. The impactors had masses ranging from $35 \mathrm{pg}$ to $0.15 \mathrm{fg}$ and speeds ranging from $2.8 \mathrm{~km} / \mathrm{s}$ to $67 \mathrm{~km} / \mathrm{s}$. The impact flash generated was measured using a photomultiplier tube and scaling laws were generated to study the dependence of observed luminosity on the mass and velocity of the impactor. Efforts to determine the mass and velocity exponents independently were successful for all targets except solar cells. The mass exponent was found to lie in the range between 0.38 and 0.64 for various targets, which
\end{abstract}

\footnotetext{
${ }^{*}$ Corresponding author. Tel.: +1 (650) 804-4689

Email address: ashish09@stanford.edu (A. Goel)

${ }^{\dagger}$ Currently at the Graduate Aerospace Laboratories of the California Institute of Technology, Pasadena, CA 91125, USA
} 
is significantly lower than the value of 1 often assumed in literature. Observations made from different angles were compared and the difference in the optical yields was found to be insignificant. The rise time of the integrated signal was found to have a negative correlation with the velocity of the impactor only for particles with speed greater than $8 \mathrm{~km} / \mathrm{s}$, which is contrary to what was observed in previous research. The correlation is however weak, with an $R^{2}$ value of 0.026 and could not be reproduced using a matched filter approach. A support vector regression based scheme was developed for estimating the velocity of the impactor, using temporal characteristics of the optical flash. The algorithm was able to estimate the velocity with a mean estimation error of $6.56 \mathrm{~km} / \mathrm{s}$.

Keywords: Optical flash, Hypervelocity impact, Meteoroid, Orbital debris, Flash, Estimation

\section{1. Introduction}

2 The solar system is abound with a variety of meteoroids and dust streams.

3 They have been detected in the interplanetary space and near other planets 4 in the solar system. In the course of its orbit around the sun, the Earth 5 itself passes through several particle streams, giving rise to meteor showers.

${ }_{6}$ The Earth is also subjected to a steady bombardment of sporadic mete7 oroids from different directions $(1,2)$. In addition to the meteoroids, Earth's 8 space environment comprises an ever growing population of orbital debris. 9 Satellites in space are hit by such meteoroids and orbital debris, collectively 10 known as hypervelocity particles, on a regular basis. It is estimated that a 11 nanogram-sized particles impacts a $1 \mathrm{~m}^{2}$ surface approximately once per day 
$(1,3)$. Traveling at relative speeds ranging from $7 \mathrm{~km} / \mathrm{s}$ in the case of orbital debris to $72 \mathrm{~km} / \mathrm{s}$ in the case of meteoroids, when these particles impact a surface, they vaporize, ionize and produce a radially expanding plasma that can trigger electrostatic discharges $(4,5,6,7,8)$. This plasma has also been found to emit electromagnetic pulses that can have detrimental effects on electrical sub-systems on the satellite $(9,10,11)$.

However, our knowledge of these particles is limited. Few dust detectors such as those on Ulysses (12) and Galileo (13) have been flown and in-situ measurements in the vicinity of the Earth have also been limited. One of the most characteristic signatures of a hypervelocity impact event is the optical flash that is generated. As the impactor deposits its kinetic energy into the target material, both the impactor and a part of the target material get heated to high temperatures, giving rise to the impact flash. Such flashes have been observed in numerous ground-based experiments $(14,15,16,17,18,19)$ and have also been associated with meteoroid impacts on the surface of the moon $(20,21,22)$. Since light reaches the sensors faster than the plasma, optical signal can provide an estimate of the time of impact and serve as a trigger for data acquisition from other sensors. More importantly, properties of the optical flash can be used to estimate different impact parameters such as the mass and velocity of the impactor, thus providing a simple yet effective way of understanding the population of hypervelocity particles near Earth, and dust particles elsewhere in the solar system.

Several measurements of the hypervelocity impact flash have been carried out at both Van de Graaff dust accelerator facilities and light gas guns. In the 1970s, G. Eichhorn presented seminal results analyzing the dependence of the 
strength of the observed signal on the impact parameters $(14,15)$. He also postulated that the rise time of the integrated signal has a strong negative correlation with the velocity of the impactor, while having no dependence on its mass. This behavior was also reported by Moser et al., albeit for a small number of meteoroid impacts on the surface of the moon (23). Experiments later carried out by Burchell et al. (16) and Serna (24) showed a much weaker dependence of rise time on velocity. There have also been several efforts to probe deeper into the physics of the impact phenomenon using spectral measurements of the impact flash at light gas guns and other facilities $(17,18,19)$. Recently, the evolution of the target temperature has also been studied for high velocity impacts at the Colorado dust accelerator facility (25).

In this paper, we describe our efforts to measure the optical emission from hypervelocity impacts and to determine the impact parameters from those measurements. Section 2 describes the experimental setup. In section 3, we present the results obtained from analysis carried out on the photomultiplier tube (PMT) data. The results fall into two main categories. First, we present results from analysis of the strength of the measured signal in sections 3.1 and 3.2. We then use temporal aspects of the signal in section 3.3 to estimate the velocity of the impactor. Our conclusions and future plans are presented in section 4 . 


\section{Experimental Approach}

\subsection{Experiment Facility}

Hypervelocity impact tests were carried out during a 2-week campaign in 2011 at the Van de Graaff dust accelerator facility, at the Max Planck Institute for Nuclear Physics in Heidelberg, Germany. The experimental facility is described in detail by Mocker et al. (26). At this facility, dust particles are charged using a Van de Graaff generator and then accelerated from a $2 \mathrm{MV}$ terminal into a vacuum chamber. The target is placed in the vacuum chamber in the path of the beam. On their way into the vacuum chamber, the particles also pass though a couple of induction loops that measure the charge on the particle. Using the charge and time difference between the incidence of the signal in the two loops, one can calculate the mass and velocity of the impactor. Due to the acceleration mechanism, the speed of a particle is tightly coupled to its mass. The smaller particles have higher charge to mass ratio and hence end up being accelerated to higher speeds.

During our experimental campaign, spherical iron projectiles were used with masses ranging from $35 \mathrm{pg}$ to $0.15 \mathrm{fg}$ and speeds ranging from $2.8 \mathrm{~km} / \mathrm{s}$ to $67 \mathrm{~km} / \mathrm{s}$. Over a duration of 8 days, a total of 7077 particles with known mass and velocity were shot at the targets; the optical sensor was used for 3720 of these impacts. Figure 1 shows a scatter plot of the masses and velocities of the projectiles for which the optical sensor was used during the experiment, along with the cumulative number of impacts as a function of impact speed. The figure clearly shows that a majority of the impacts were at speeds lower than $10 \mathrm{~km} / \mathrm{s}$. All impacts were from an angle of 30 degrees 

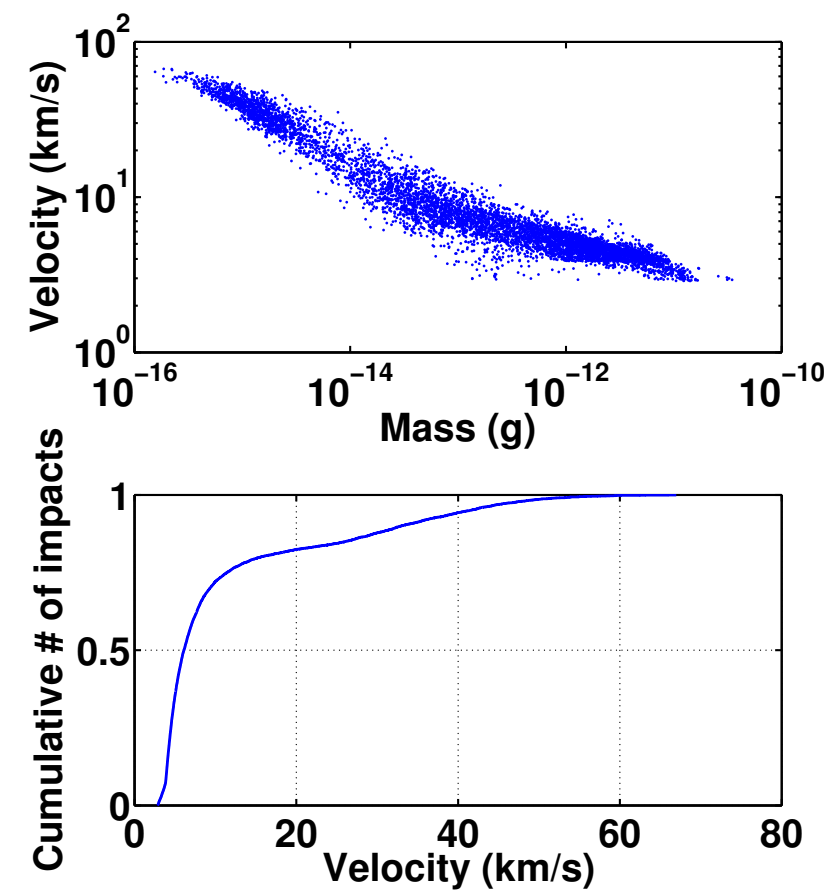

Figure 1: (a) Scatter plot of 3720 separate impact events demonstrating the coupling between mass and velocity (b) Cumulative number of impacts as a function of impact velocity.

below the target normal.

\subsection{Impact Targets}

Various targets comprising bare metal surfaces and spacecraft materials were used. These targets and their abbreviated names which will henceforth be used in this paper are listed in Table 1. For the sizes of particles shot, these targets were too thick for the particles to penetrate through. Visual investigation of the target surfaces before and after the experimental campaign revealed no signs of damage. The targets were also examined using an electron microscope and showed no impact-crater at the micron level. At dif- 
Table 1: Targets used during the experimental campaign.

\begin{tabular}{|c|c|}
\hline Target material & Abbreviation \\
\hline \hline Tungsten & TGT-W \\
\hline Aluminum & TGT-Al \\
\hline Solar Panel Substrate & LMSP \\
\hline Solar cells used in GEO & LMSC-GEO \\
\hline Solar cells used in LEO & LMSC-LEO \\
\hline Optical Solar Reflector (standard) & LMOSR-Std \\
\hline Optical Solar Reflector (conductive) & LMOSR-Con \\
\hline Copper & SRIF-Cu \\
\hline
\end{tabular}

ferent times during the experiment, the targets were positively or negatively biased to simulate the effects of spacecraft charging. However, we do not expect these biases to have any impact on observations of the impact flash. A description of the various target surfaces can be found in Lee et al.(6).

\subsection{Sensor}

A host of different sensors were deployed to measure the optical, plasma and RF emission from the impacts. A description of the plasma and RF sensors and results from analysis of the data collected by them can be found in Lee et al. (6) and Close et al. respectively (9). In this paper, we focus on results from the Hamamatsu H10721-110 photomultiplier tube used for optical measurements. The PMT has maximum responsivity in the visible wavelength band. Important specifications of the sensor are listed in Table 2. The impact flash typically has a duration on the order of microseconds 
Table 2: Specifications of the PMT.

\begin{tabular}{|c|c|}
\hline Dimensions & $50 \mathrm{~mm} \mathrm{X} 22 \mathrm{~mm} \mathrm{X} 22 \mathrm{~mm}$ \\
\hline Active Diameter & $8 \mathrm{~mm}$ \\
\hline Responsivity & $2.2 \times 10^{5} \mathrm{~A} / \mathrm{W}$ \\
\hline Dark Current & $1 \mathrm{nA}$ \\
\hline Rise Time & $0.57 \mathrm{~ns}$ \\
\hline Wavelength range & $230-700 \mathrm{~nm}$ \\
\hline
\end{tabular}

and in order to retain the temporal characteristics of the signal, the current output of the sensor was amplified and converted into a voltage signal using an op-amp based transimpedance amplifier. Three different plasma sensors were used during the experiment and since the mounting of the PMT was tied to the mounting of the plasma sensor, the PMT was also mounted in three different geometrical configurations. The distance of the sensor from the point of impact and the angle between the axis of the sensor and the target normal for these configurations are listed in Table 3. Figure 2 shows the sensor mounted in three different configurations in the chamber, and figure 3 contains a sketch of the beam line, target normal and the axis of the sensor for the three different configurations.

\section{Results and Discussion}

An optical flash was observed for a considerable fraction of impacts, especially at high velocities. Figure 4 shows the number of impacts for each of the targets used and the fraction of those impacts for which an optical flash was observed. Note that this graph is not a true representation of the 
Table 3: Position and angle of the PMT for three different configurations.

\begin{tabular}{|c|c|c|}
\hline Configuration & $\begin{array}{c}\text { Distance from point of } \\
\text { impact }(\mathbf{c m})\end{array}$ & $\begin{array}{c}\text { Angle from target } \\
\text { normal (degrees) }\end{array}$ \\
\hline \hline 1 & 11.0 & 20.0 \\
\hline 2 & 11.5 & 8.5 \\
\hline 3 & 14.0 & 39.0 \\
\hline
\end{tabular}
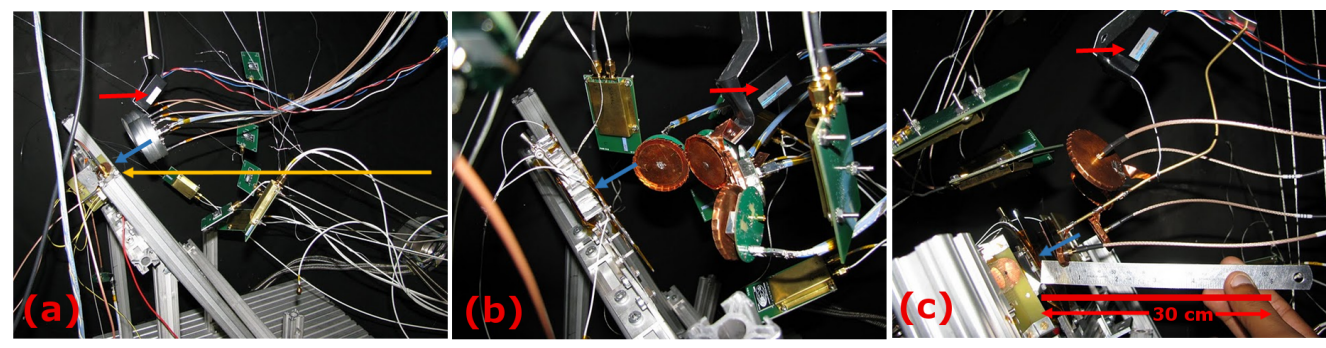

Figure 2: Three different geometrical configurations used during the experiment campaign. In these images, the red arrow points at the PMT, the blue arrow points at the target and the yellow arrow indicates the direction in which the particle comes and impacts the target. (a) Configuration 1 with the PMT 20 degrees above target normal (b) Configuration 2 with the PMT 8.5 degrees above target normal (c) Configuration 3 with the PMT 39 degrees above target normal. 


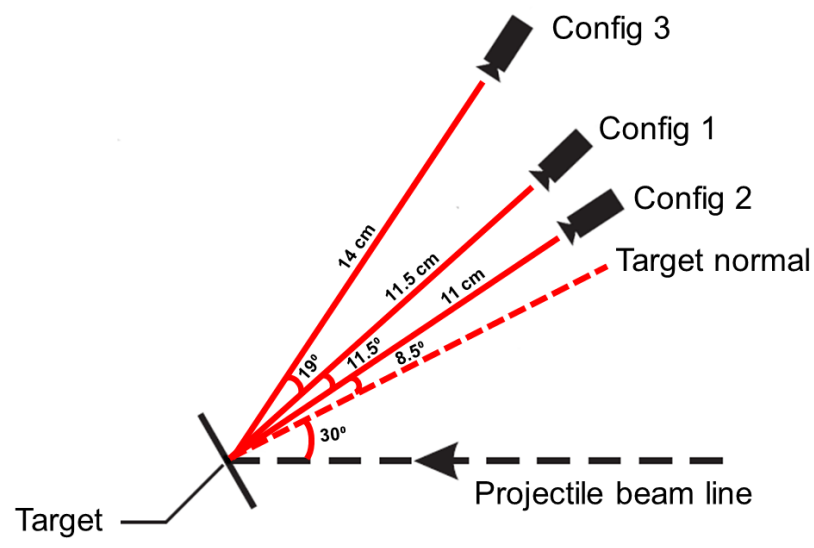

Figure 3: A drawing showing the positions of the PMT with respect to the target normal for three different geometrical configurations used.

likelihood of a target to produce a flash strong enough to be detected by our sensor. The dust accelerator facility has a strong tendency to produce faster particles in the first few days of operation after the installation of a new source. Since different targets were used at different times during the experiment, the detection rates in figure 4 are biased by the mass and velocity distribution that different targets were subjected to. This can be seen clearly from figure 5, which shows that for particles with speed in excess of $15 \mathrm{~km} / \mathrm{s}$, the optical detection rate is close to $90 \%$ and hence the overall low detection rates come from the fact that most of the impacts were at low velocities, as shown in figure 1b. From figure 4, one can also see that a majority of the impacts and detected flashes were from the tungsten target while no flashes were observed from the aluminum target. Hence most of our analysis in the following sections will be performed with the data from impacts on tungsten. The absence of plasma signal from aluminum target was also observed by Lee et al. and might be due to the low density and 


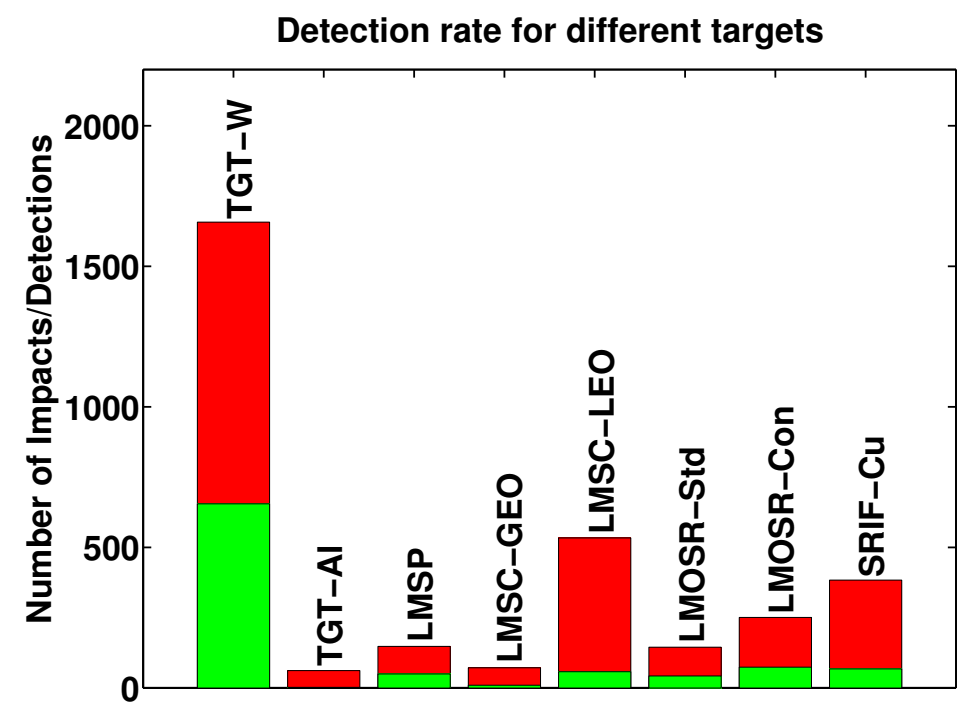

Figure 4: Detection rate for different targets. The height of the green section indicates the number of impacts out of total (red) for which an impact flash signal could be detected above the noise floor. Note that this graph only includes the impacts for which the photomultiplier tube was used.

high malleability of the aluminum target. Figure 6a shows the optical signal from a typical impact event. We refer to the region marked in red as a peak. Almost all the observed signals are in the form of a single peak with a sharp rise followed by an exponential decay.

\subsection{Scaling Laws}

\subsubsection{Estimation of Mass and Velocity Exponents}

The measured strength of the optical signal $(P)$ is known to depend exponentially on the mass $(m)$ and velocity $(v)$ of the impactor according to the following equation $(14,15)$

$$
P=K m^{\alpha} v^{\beta}
$$




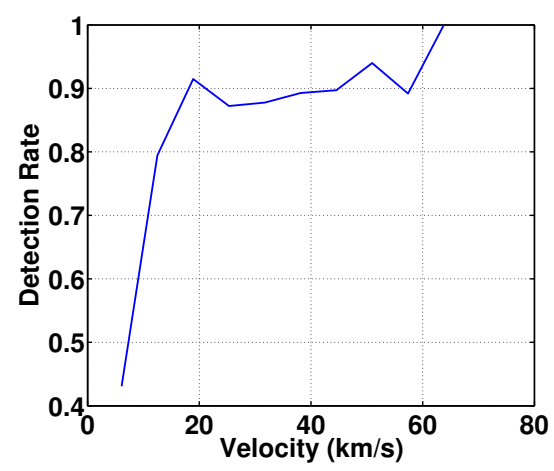

Figure 5: Detection rate as a function of the velocity of the impactor. Non overlapping bins of width $5 \mathrm{~km} / \mathrm{s}$ were used for generating this plot.
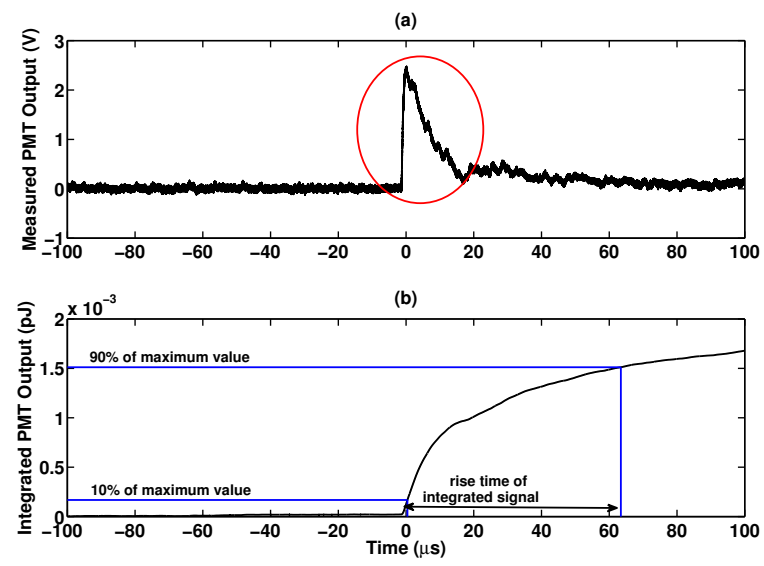

Figure 6: (a) A typical signal showing a sharp rise followed by an exponential drop-off. This signal was measured from the impact of a $1.51 \mathrm{fg}$ particle on tungsten target at 44.4 $\mathrm{km} / \mathrm{s}$. The peak is marked by the red outline. (b) Time-integrated version of the signal in (a). The rise time is calculated as the time-difference between the $10 \%$ and $90 \%$ points. 
Here $K$ represents the optical yield of the material whereas $\alpha$ and $\beta$ are the mass and velocity exponents respectively. Expressing this equation in the logarithmic space turns it into a linear equation. For each of the impacts on a given target, we can write this linear equation to end up with a system of linear equations as shown below

$$
\begin{aligned}
\log P_{1} & =\log K+\alpha \log m_{1}+\beta \log v_{1} \\
\log P_{2} & =\log K+\alpha \log m_{2}+\beta \log v_{2} \\
\vdots & \\
\log P_{n} & =\log K+\alpha \log m_{n}+\beta \log v_{n}
\end{aligned}
$$

This can be written as $P=A x$, where

$$
P=\left[\begin{array}{c}
\log P_{1} \\
\log P_{2} \\
\vdots \\
\log P_{n}
\end{array}\right] \quad A=\left[\begin{array}{ccc}
1 & \log m_{1} & \log v_{1} \\
1 & \log m_{2} & \log v_{2} \\
\vdots & & \\
1 & \log m_{n} & \log v_{n}
\end{array}\right] \quad x=\left[\begin{array}{c}
\log K \\
\alpha \\
\beta
\end{array}\right]
$$

We end up with one such system of equations for each target and an obvious next step is to compute the least squares estimate of $\alpha$ and $\beta$. However, as shown in figure 1a, the masses and velocities of the projectiles are not independent of each other. Consequently, the condition number of the $A$ matrix is close to 230 for the entire dataset, which significantly reduces the accuracy of the least squares estimate. We used the maximum height of the peak as a measure of the optical signal strength and only in the case of the tungsten target we were able to arrive at robust estimates of the mass and velocity exponents due to the large number of impacts on that target. Table 
Table 4: Values of $\alpha$ and $\beta$ obtained for different targets using least squares (linear fit)

\begin{tabular}{|c|c|c|c|c|}
\hline Target material & $\alpha$ & p-value & $\beta$ & p-value \\
\hline \hline TGT-W & $0.64 \pm 0.09$ & $4.2 \mathrm{e}-34$ & $2.74 \pm 0.32$ & $1.1 \mathrm{e}-47$ \\
\hline LMSP & $0.42 \pm 0.28$ & 0.0043 & $1.35 \pm 0.82$ & 0.0017 \\
\hline LMSC-GEO & - & $>0.05$ & - & $>0.05$ \\
\hline LMSC-LEO & - & $>0.05$ & - & $>0.05$ \\
\hline LMOSR-Std & $0.53 \pm 0.24$ & $7.7 \mathrm{e}-5$ & $1.32 \pm 0.66$ & $2.2 \mathrm{e}-4$ \\
\hline LMOSR-Con & $0.41 \pm 0.20$ & $1.4 \mathrm{e}-4$ & $1.08 \pm 0.56$ & $2.5 \mathrm{e}-4$ \\
\hline SRIF-Cu & $0.38 \pm 0.20$ & $3 \mathrm{e}-4$ & $0.86 \pm 0.53$ & 0.0017 \\
\hline
\end{tabular}

4 lists the mass and velocity exponents computed for each target, along with the $p$-value corresponding to those estimates. Note than a value of $p>0.05$ indicates a failure to reject the null hypothesis that the optical power is uncorrelated with the mass and velocity of the projectile.

In previous work done by Eichhorn, the author chose a subset of impacts with similar projectile velocities to compute the mass exponent $(14,15)$. Such an estimate is highly non-robust since it uses only a fraction of the information available in the dataset. The estimates are bound to depend significantly on the specific subset chosen to compute the exponent.

From Table 4, we find that the mass exponent for different targets has a value close to 0.5 and the confidence interval in all those cases excludes the value of 1 , which has often been the value assumed in previous work (16). When studying meteoroid impacts on the lunar surface, luminous efficiency (ratio of optical yield and kinetic energy) is also treated only as a function 
of velocity and not as a function of mass (27). Our data suggests that the velocity exponent is also significantly lower than the range of values (2.54) often quoted in literature $(14,15,16,18)$.

We would like to note here that these scaling laws are purely empirical and primarily serve an engineering purpose to get an estimate of how the signal strength would scale when looking at different mass and velocity regimes. The non-integer exponents thus obtained are a result of simplifying the complicated physical processes occurring during the impact and expressing it in the form of a simple power law. Hence if the velocity exponent is found to be varying over a large range of values, there is no reason to expect the mass exponent to have a nice integer value of 1 .

An alternative to using the peak height of the observed signal (shown in figure 6a) is to use the total optical energy, which can be estimated by calculating the maximum height of the integrated signal. The integrated signal is obtained by integrating over time, the measured signal shown in figure $6 \mathrm{a}$ and this integrated signal can be seen in figure $6 \mathrm{~b}$. The maximum height of the integrated signal now represents the total area under the light curve and is a measure of the total optical energy released during the flash. Using this metric for the tungsten target, the mass exponent was found to be $0.60 \pm 0.13$ and the velocity exponent was found to be $2.76 \pm 0.46$. Comparing these numbers with the ones obtained using the peak height, we find that the difference in the estimates of the exponents is smaller than the errors in the estimates. Hence we were able to arrive at similar estimates for the mass and velocity exponents using two different metrics for the strength of the optical signal. 
As mentioned earlier, when studying the optical emission from meteoroid ablation and meteoroid impacts on the lunar surface, the optical yield is usually plotted as a function of the kinetic energy of the incoming particle to estimate the luminous efficiency or the fraction of kinetic energy that goes into optical emission. In our case, since the peak height of the signal and the total optical energy are so strongly correlated, the dependence of total optical energy on the kinetic energy of the particle can be inferred from the scaling laws. Since our scaling laws do not necessarily predict a mass exponent of 1 and a velocity exponent of 2 , the luminous efficiency will depend on both mass and velocity. Figure 7 plots the total optical energy as a function of the kinetic energy of the impactor for impacts on tungsten. Note that in this plot, we have accounted for the solid angle subtended by the sensor at the point of impact and normalized assuming that the emission is isotropic in the hemisphere above the target. We shall see in section 3.2 that this may not be necessarily true. However, this assumption is sufficient to arrive at rough estimates of the luminous efficiency. From the plot, one can see that in general, the higher the kinetic energy of the impactor, higher is the optical yield, although this trend does not hold for the few particles with very high kinetic energy. We can also see that in most cases, less than a thousandth of the kinetic energy goes into optical emission in the visible band.

\subsubsection{Estimation of Velocity Exponent for Linear Mass Dependence}

In order to compare our results with previous efforts in this area, we assumed a linear mass dependence $(\alpha=1)$, normalized the observed signal strength by the mass of the impactor and estimated the velocity exponents for the different targets. Table 5 lists the values of $\beta$ obtained, along with 


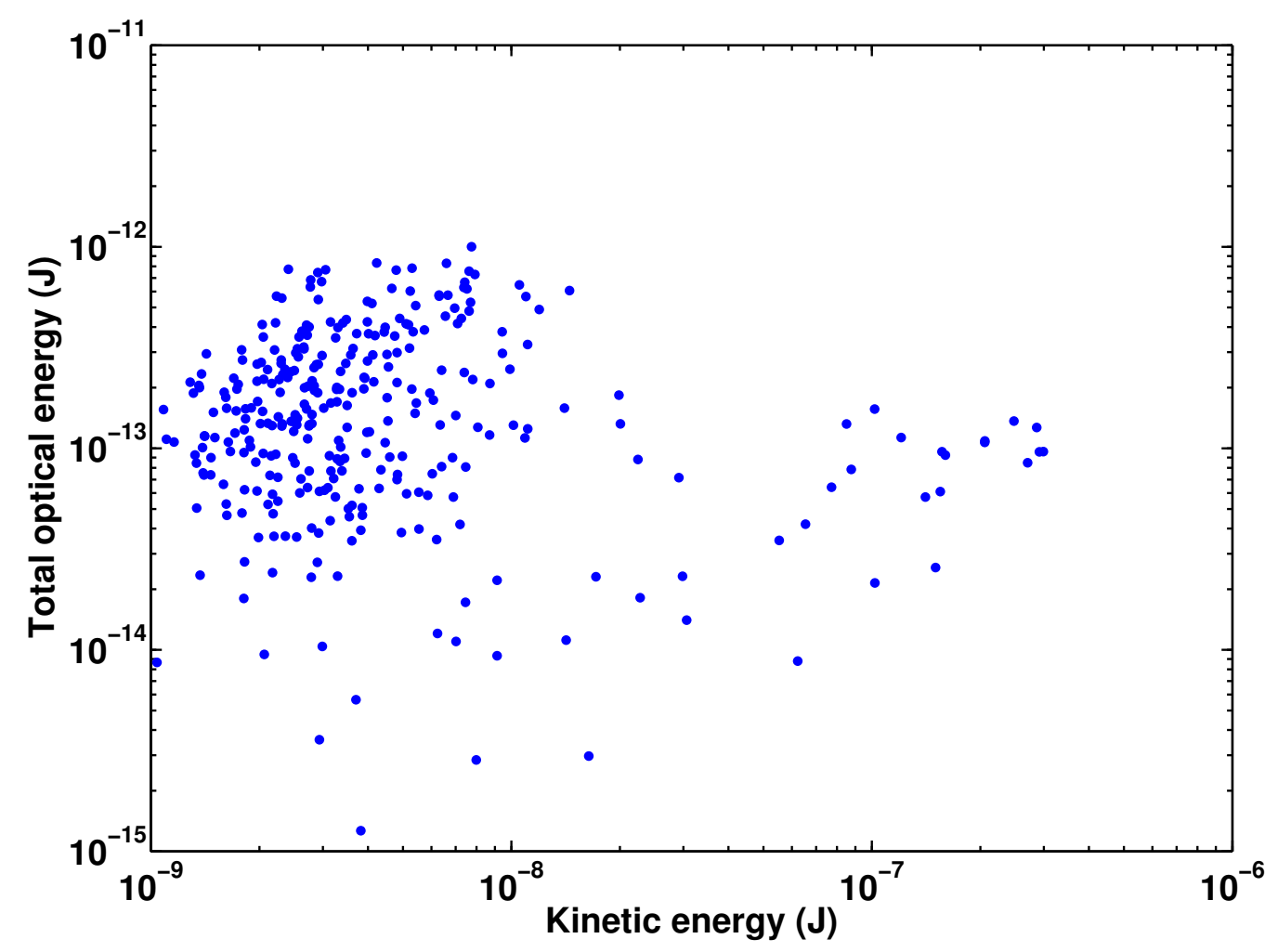

Figure 7: Dependence of total optical energy emitted on the kinetic energy of the impactor for impacts on tungsten target. 
Table 5: Values of $\beta$ obtained for different targets using least squares (linear fit) on data normalized by the mass of the impactor.

\begin{tabular}{|c|c|c|}
\hline Target material & $\beta$ & p-value \\
\hline \hline TGT-W & $3.79 \pm 0.08$ & $2.3 \mathrm{e}-232$ \\
\hline LMSP & $2.96 \pm 0.26$ & $8.1 \mathrm{e}-24$ \\
\hline LMSC-GEO & $2.36 \pm 0.36$ & $1.9 \mathrm{e}-6$ \\
\hline LMSC-LEO & $2.85 \pm 0.25$ & $1.3 \mathrm{e}-25$ \\
\hline LMOSR-Std & $2.69 \pm 0.33$ & $5.3 \mathrm{e}-17$ \\
\hline LMOSR-Con & $2.57 \pm 0.22$ & $5.6 \mathrm{e}-30$ \\
\hline SRIF-Cu & $2.41 \pm 0.22$ & $8.4 \mathrm{e}-28$ \\
\hline
\end{tabular}

the $95 \%$ confidence interval and the $p$-value. Figure 8 shows the linear fit for different target materials. We observe that the values of $\beta$ now obtained are significantly higher and in the case of tungsten for which there is previous data available, our value of $\beta$ agrees with the numbers that were obtained by others making the $\alpha=1$ assumption $(14,15,16,18)$. Also, since we now fit for one less parameter, we can obtain estimates of $\beta$ for all the targets with high statistical significance. The higher significance level is also due to the fact that the mass and velocity of the impactor are strongly related, as shown in figure 1a. Hence normalizing with respect to the mass introduces a spurious correlation with velocity. This however cannot account entirely for the large exponent values listed in Table 5 and hence the values of $\beta$ obtained have partial contributions from a true dependence of signal strength and a systematic dependence of the normalizing factor (mass) on velocity. 

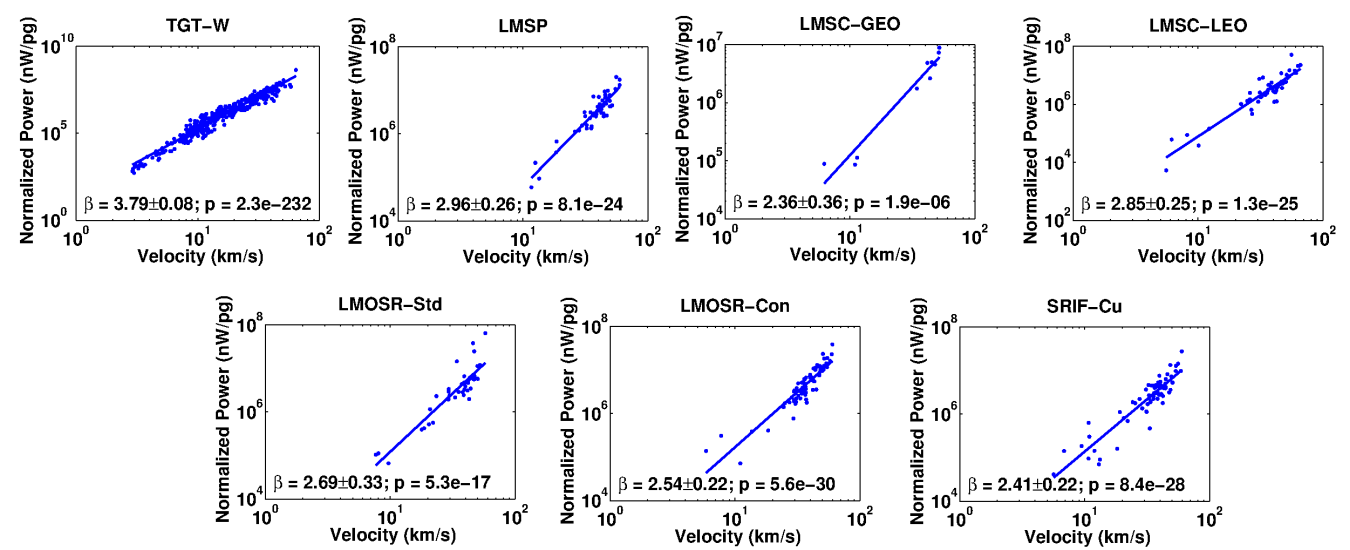

Figure 8: Linear fits to the normalized impact flash data for different targets. The values of $\beta$ obtained using the fits are also shown on the plot.

\subsection{Dependence on Observation Angle}

When interpreting results from different experimental setups, it is important to account for the variability introduced by the geometry of the setup. As discussed in section 2.3, the sensor was used in 3 different configurations. For configuration 2, we did not have enough data to estimate the mass and velocity exponents simultaneously. We hence normalized the signal strength by $m^{\alpha}$ where $\alpha$ was chosen to be 0.64 , based on Table 4 . We further normalized these results by the square of the distance of the sensor from the point of impact. This allows us to eliminate the effect of the $1 / r^{2}$ fall-off and study the effect of observation angle on the strength of measured signal. The values obtained for the velocity exponent $\beta$ are listed in Table 6 along with the confidence intervals and $p$-values. Note that the three different configurations are compared only for impacts on tungsten target. Figure 9 also shows the fits for the three different configurations. We find that the velocity exponent remains approximately the same for the three geometries. We hence use the 
Table 6: Fitting parameters for different geometric configurations assuming same mass exponent (tungsten target). The yield is computed assuming the same velocity exponent of 2.61 .

\begin{tabular}{|c|c|c|c|}
\hline Configuration & $\beta$ & p-value & Yield (K) \\
\hline \hline 1 & $2.61 \pm 0.08$ & $7.2 \mathrm{e}-184$ & $(11.8 \pm 7.7) \mathrm{e} 9$ \\
\hline 2 & $2.64 \pm 0.19$ & $2.0 \mathrm{e}-16$ & $(7.5 \pm 3.2) \mathrm{e} 9$ \\
\hline 3 & $2.84 \pm 0.13$ & $1.1 \mathrm{e}-104$ & $(6.9 \pm 3.0) \mathrm{e} 9$ \\
\hline
\end{tabular}

same value of $\beta$ for the three configurations to compute the yields listed in Table 6. The yield is highest for configuration 1 with the sensor at an angle of 20 degrees from the target normal and lower for 8.5 and 39 degree angles suggesting that variation of yield with angle is not monotonic. A similar trend has been observed when looking at the angular distribution of ejecta and the corresponding flash from secondary impacts (28) but no such trend has been reported for flash directly from the impact. Since the impacts were from an angle of 30 degrees below the target normal, the crater formation and temperature distribution in the impact zone were probably asymmetric, which could have lead to the observed asymmetry in the optical emission. However, the errors in these yield estimates are quite large and the mean value for each configuration lies within the confidence interval of the mean value for the other configurations. Hence we cannot rule out the possibility of there being no significant dependence of optical yield on the observation angle. 


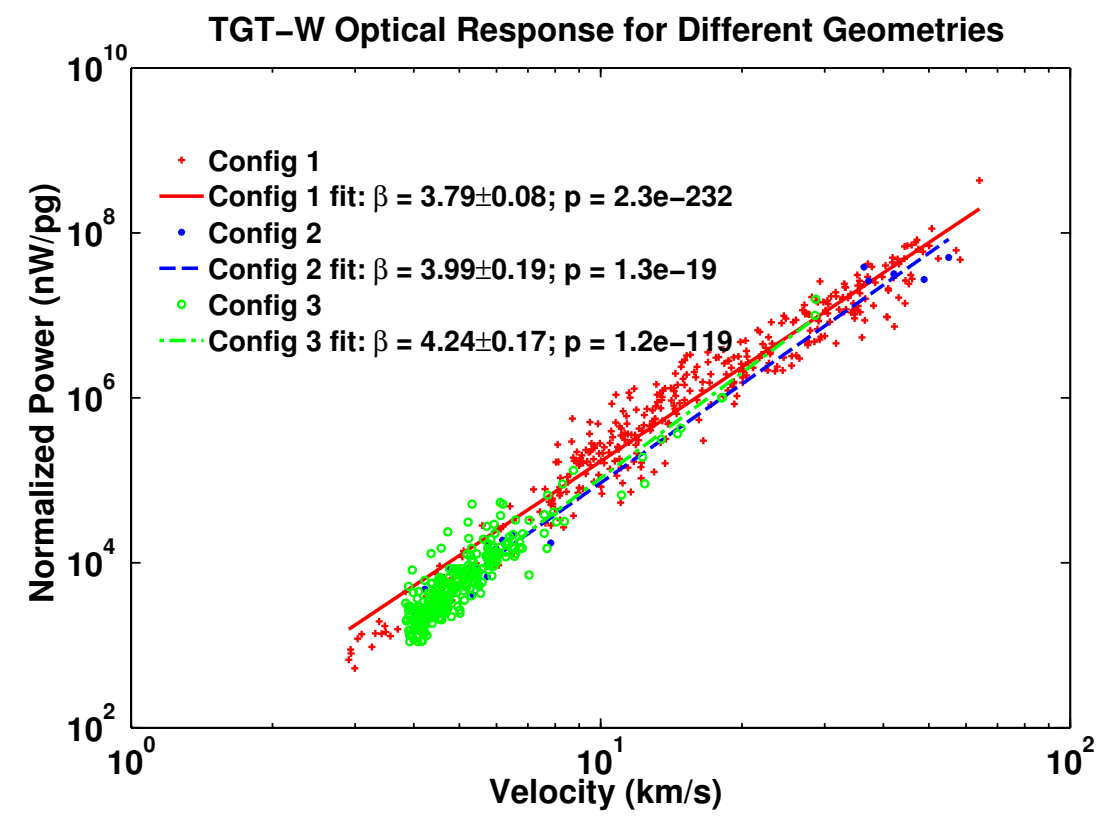

Figure 9: Linear fits to data normalized by the mass of the impactor for three different geometrical configurations. 


\subsection{Dependence of Rise Time on Velocity}

Our analysis in section 3.1 shows the dependence of optical power on the mass and velocity of the impactor and the angle of observation. This however does not allow us to decouple the mass from the velocity, and hence we need a method to estimate at least one of these parameters independently from the other. In this section we describe how the temporal evolution of the optical signal can be used to estimate the velocity.

\subsubsection{Rise Time of Integrated Signal vs Velocity}

In the mid 1970s, Eichhorn had postulated that the rise time of the integrated signal has a strong negative correlation with the velocity of the impactor but no correlation with the mass $(14,15)$. Burchell et al. (16) and Serna (24) found the correlation to be much weaker but did not quantify the strength of the correlation, if any. In order to verify this, we also started off by studying the rise time of the integrated signal. In recent experiments carried out at the Colorado dust accelerator facility, the temperature evolution of the impact zone was measured using multi-spectral measurements of the flash (25). Based on those results and results from similar experiments using photodiodes at a light gas gun facility (18), we expect the rise time of the integrated signal or the total duration of the observed signal to be on the order of 10 microseconds.

When looking at the temporal characteristics of any signal, one needs to account for temporal distortions created by the amplification and data collection units. The frequency response of the transimpedance amplifier used to amplify the output of the PMT was measured, and the transfer function of the amplifier thus obtained was used to remove the effect of 
the amplifier from the observed waveforms. Since the amplifier has limited bandwidth, the performance of the amplifier falls off at high frequencies and in the process of inversion, creating high-frequency noise in the deconvolved signal. This limits our ability to obtain the true temporal characteristics of the signal. But since the bandwidth of the amplifier is close to $10 \mathrm{MHz}$, errors introduced in the measurement of microsecond-duration signals are expected to be small

For each of the impacts on tungsten, the $10 \%-90 \%$ rise time of the integrated signal $\left(t_{\text {int }}\right)$ was calculated, as shown in figure $6 \mathrm{~b}$. These values are plotted against the velocity $(v)$ of the impactor in figure 10. This figure shows that for velocities less than $8 \mathrm{~km} / \mathrm{s}$, there is no significant correlation between the rise time and velocity. However, for velocities beyond 8 $\mathrm{km} / \mathrm{s}$, there is a weak trend of rise time decreasing with increasing velocity. In fact, there appears to be a sharp transition in the hypervelocity impact phenomenon around $8-10 \mathrm{~km} / \mathrm{s}$. In addition to figure 10, this sharpness in transition can be seen in figure 5 and has also been reported in the case of electromagnetic pulse detection by Close et al. (9). Fletcher et al. (5) attribute this to the transition from a state of partial ionization to complete ionization in the impact region. For our analysis, we restricted ourselves to data points beyond $8 \mathrm{~km} / \mathrm{s}$ and fit a linear model to the data. The fitting parameters and the values of metrics measuring the goodness of the fit are listed in Table 7 . While the correlation between $t_{\text {int }}$ and $v$ is statistically significant, the very low value of $R^{2}$ shows that a linear model does not do a great job of explaining the observed trend.

Eichhorn had postulated that the relationship between $t_{i n t}$ and $v$ is better 


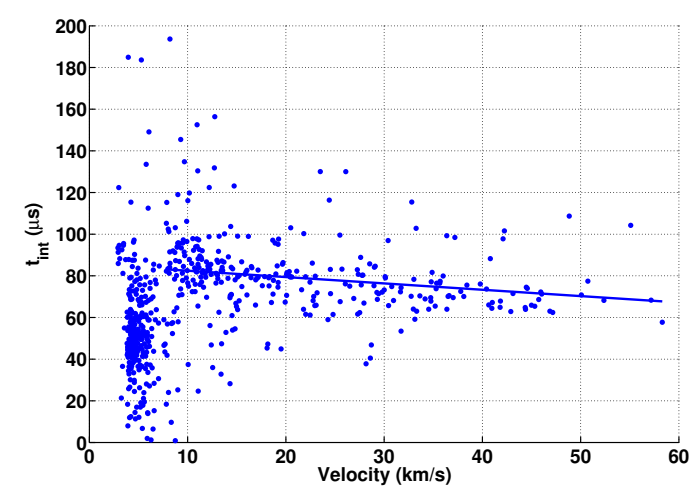

Figure 10: Relationship between the $10 \%-90 \%$ rise time of the integrated signal and the velocity of the impactor. The straight line represents the linear fit for velocities greater than $8 \mathrm{~km} / \mathrm{s}$. The data in this figure is only for impacts on tungsten target.

described in the form of a power law $(14,15)$. Hence we also analyzed the relationship between $\log \left(t_{\text {int }}\right)$ and $\log (v)$. The correlation was found to be statistically insignificant in the logarithmic space, which is probably due to the large intercept in the relationship between $t_{\text {int }}$ and $v$. The integrated signal's rise time was found to have no correlation with the mass of the impactor. We also looked for correlation in the case of the target with the second highest number of impacts (conductive OSR) but due to the relatively small number of impacts, correlation was observed neither with mass nor with the velocity of the impactor.

\subsubsection{Matched Filter}

The motivation behind looking at the rise time of the integrated signal is to use it as a measure of the duration of the flash. Since the low value of $R^{2}$ suggested that $t_{\text {int }}$ would not serve as a good estimator of impact velocity, matched filtering was used an an alternative for estimating the duration of 
the optical flash. Most signals follow a well-defined pattern containing an almost instantaneous rise (usually less than $2 \mu \mathrm{s}$ ) followed by an exponential fall-off as shown in figure 6a. Hence a simple function $f(t)$ was used to model the signal

$$
f(t)= \begin{cases}0 & \text { for } t<t_{0} \\ M e^{-\frac{t-t_{0}}{\tau}} & \text { for } t \geq t_{0}\end{cases}
$$

where $M$ is the maximum height, $t_{0}$ is the starting time of the signal and $\tau$ is the time constant of the exponential. We carried out a grid search to find the values of $M, t_{0}$ and $\tau$ that best describe the measured waveform. The dust accelerator facility gives us an estimate of $t_{0}$, allowing us to narrow our search region. Figure 11 shows the result of the matched filtering process for one of the impacts on tungsten. We then use the optimum value of $\tau$ as a proxy for the duration of the flash. Note that this procedure was only applied for signals stronger than $300 \mathrm{mV}$ since weak signals do not exhibit the exponential structure on a regular basis. In figure 12, we plot the time constants against the velocity of the impactor. We find that there is no correlation for velocities less than $20 \mathrm{~km} / \mathrm{s}$ and while there appears to be a negative correlation for velocities greater than $20 \mathrm{~km} / \mathrm{s}$, an attempt at fitting a linear model fails the statistical significance test with a $p$-value of 0.06 . Looking for a linear relationship in the logarithmic space fails to improve the significance level. As in the case of $t_{i n t}$ vs $v$, there exists no correlation between the time constant of the exponential and the mass of the impactor. 


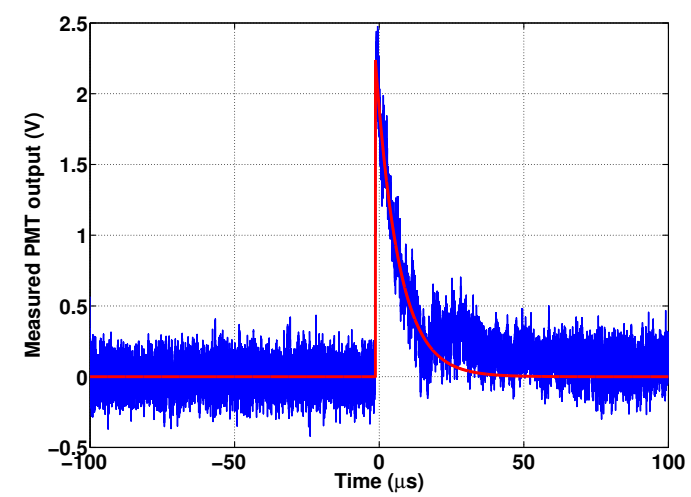

Figure 11: The curve in red shows the best exponential fit to the measured signal in blue. This waveform is for the same impact shown in figure 6 .

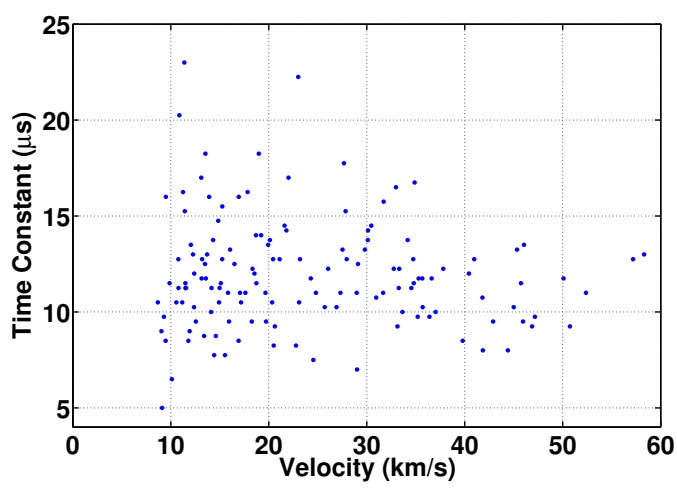

Figure 12: Time constant of the exponential decay obtained from matched filtering versus the velocity of the impactor for impacts on tungsten target. 


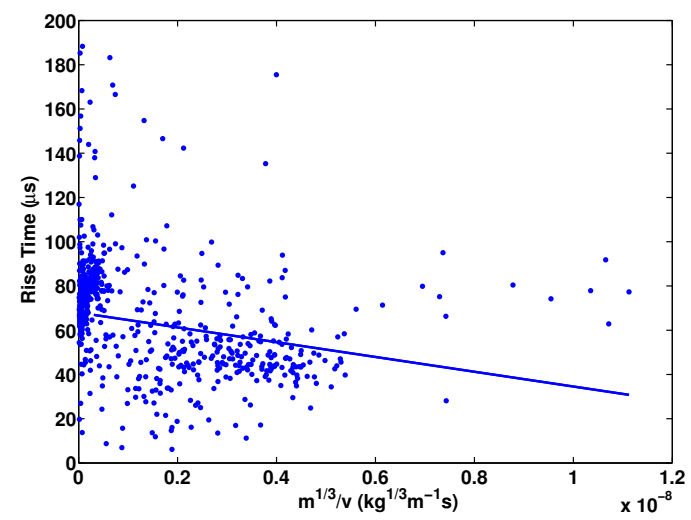

Figure 13: Relationship between the rise time of the integrated signal and Q. The straight line represents the linear fit for values of $\mathrm{Q}>3 \mathrm{e}-10$.

\subsubsection{Dimensional Approach}

In the absence of a deep physical insight into the relationship between the rise time of the integrated signal and the velocity of the impactor, one approach is to use dimensional analysis to find the right quantity that the rise time of the integrated signal would depend on. The quantity $m^{1 / 3} / v$ has the same dimensions as $\rho^{1 / 3} t_{i n t}$ and for all the tungsten impacts, since the material and hence the density $(\rho)$ of the impactor and the target are the same, we can study the relationship between $t_{i n t}$ and $m^{1 / 3} / v$. We shall refer to this quantity $m^{1 / 3} / v$ as 'Q'. Figure 13 plots $t_{i n t}$ as a function of Q. For small values of $\mathrm{Q}$, we see a clustering of data points, similar to the clustering observed for low speed impacts in figure 10. We hence restrict ourselves to impacts with $\mathrm{Q}>3 \mathrm{e}-10$ and carry out a linear fit. The fitting parameters are listed in Table 7. The correlation is found to be significant and while the value of $R^{2}$ is slightly higher than that in the case of $t_{\text {int }}$ vs $v$, the correlation is still weak. 
To summarize these findings, while there is a weak correlation between the rise time of the integrated signal and the velocity of the impactor for high speed impacts, we were unable to reproduce the correlation using the matched filter approach. This indicates that the duration of the signal is not a robust estimator of the impact velocity.

\subsubsection{Rise Time vs Velocity}

While there have been studies on the possible relationship between the rise time of the integrated signal and the velocity of the impactor, there has not really been a theoretical analysis postulating why such a relationship should exist. Detailed analysis of the impact phenomenon requires hydrocode simulations, but intuitively, the faster the projectile, the faster the rate at which it deposits energy into the target, raising its temperature and causing it to emit optical radiation. One would hence expect the rise time of the observed signal (shown in figure 6a) and not the rise time of the integrated signal (shown in figure 6b) to depend on the velocity of the impactor. We investigated the relationship between the rise time of the signal $\left(t_{r}\right)$ and the velocity $(v)$ of the impactor. The result is shown in figure 14. While a linear fit yields a significant correlation with $p$-value of 0.007 , the $R^{2}$ value of 0.026 indicates that the rise time of the signal is also not a very robust estimator for the velocity of the impactor. These fitting parameters are listed in Table 7. No correlation was observed between $\log \left(t_{r}\right)$ and $\log (v)$ or between $t_{r}$ and the mass of the impactor. 


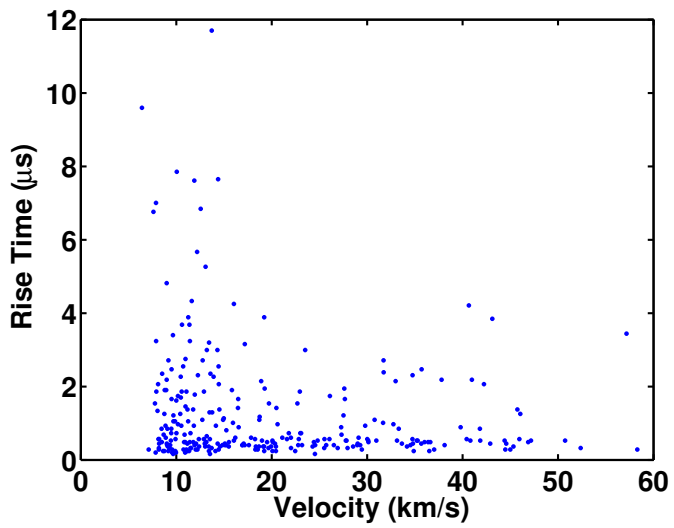

Figure 14: Rise time of the measured signal vs velocity of the impactor for tungsten target. The y-axis represents the duration over which the sharp rise in figure 6a takes place. The straight line shows a linear fit to the data.

Table 7: Parameters of linear fit between different measures of the temporal characteristics of the signal and different combinations of impact parameters. Note that the p-value listed is associated with the slope and not the intercept.

\begin{tabular}{|c|c|c|c|c|c|}
\hline x-variable & y-variable & slope & intercept & p-value & $R^{2}$ \\
\hline$t_{\text {int }}$ & $\mathrm{v}$ & $-0.30 \pm 0.19$ & $85.5 \pm 4.6$ & 0.002 & 0.03 \\
\hline$t_{i n t}$ & $\mathrm{Q}\left(m^{1 / 3} / v\right)$ & $(3.3 \pm 1.4) e 9$ & $68.0 \pm 4.4$ & $7 \mathrm{e}-6$ & 0.05 \\
\hline$t_{r}$ & $\mathrm{v}$ & $-0.023 \pm 0.016$ & $1.70 \pm 0.37$ & 0.007 & 0.026 \\
\hline
\end{tabular}




\subsection{Data Driven Approach to Estimation of Impact Parameters}

As discussed in section 3.3.4, the choice of using rise time of the integrated signal appears to be a convenient heuristic rather than a metric borne out of impact physics. There could very well be other temporal characteristics of the signal that are heavily dependent on the velocity of the projectile. In the absence of deep physical insight into how the impact velocity influences the time evolution of the optical signal, one powerful approach is to create a data-based model. We use a technique developed in the late 90s known as support vector regression (SVR) (29). This method is similar to the wellknown machine learning tool known as support vector machine but instead of just classifying data into a fixed number of classes, SVR builds a model using training data and then provides a real-valued estimate of the unknown parameter for new test data. Especially when using infinite dimensional kernels such as a Gaussian kernel, the algorithm itself figures out which temporal characteristics of the signal are relevant in estimating the unknown parameter (velocity) and ignores the rest.

In order to implement this method, we used the open source toolbox LIBSVM (30). For each waveform from a tungsten target impact, a 62dimensional feature vector was created. The maximum height of the signal and the rise time of the integrated signal were used as the first two components of the feature vector. Using the matched filter technique described in section 3.3, the starting point of each signal was identified and the waveform was then normalized by its maximum height. The remaining 60 dimensions of the feature vector were populated with the mean values of this normalized waveform in 60 bins spanning a fixed duration of $50 \mu \mathrm{s}$. The bins were more 


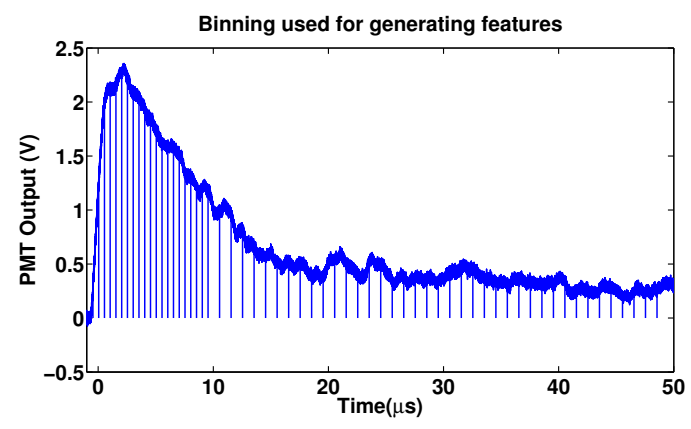

Figure 15: Edges of bins used for generating feature vectors for support vector regression.

densely packed close to the beginning of the signal since we expect the initial part of the signal to be more heavily influenced by the speed of the impactor, as discussed in section 3.3.4. The edges of the bins used for a sample peak can be seen in figure 15 .

Each component of the feature vectors thus obtained was then normalized across all impacts such that after normalization, the components had values between 0 and 1 . The normalized data were then used to determine the optimum values of parameters $C$ and $\gamma$ for the infinite-dimensional, radial basis function kernel. Having chosen the best $C$ and $\gamma$, randomly chosen $80 \%$ of the feature vectors were used for training the model with known values of impact velocity and the remaining $20 \%$ of the data were used for testing. The predicted velocity values were compared with true velocities to arrive at an error estimate. This process of validation was then repeated 1000 times, each time with randomly chosen training and test data sets; the error estimates were then averaged to arrive at a more robust metric for the performance of the algorithm. On account of the averaging over 1000 instances, the result was not sensitive to the $80-20$ ratio of training and test data. The results were 
Table 8: Model selection table showing estimation error for different subsets of feature vectors

\begin{tabular}{|c|c|}
\hline Features used & Mean Error $(\mathbf{k m} / \mathbf{s})$ \\
\hline \hline All 62 & 6.56 \\
\hline Only max. height and rise time & 8.08 \\
\hline All except max. height and rise time & 6.79 \\
\hline
\end{tabular}

also found to not change significantly by increasing the number of iterations over which the averaging was carried out.

For one of these cross-validation tests, the predicted values are plotted against the true velocities in figure 16 . The performance results for different combinations of the feature vectors are listed in Table 8. The table shows that there is a significant improvement in performance of the algorithm when using other temporal aspects of the signal, in addition to the rise time of the integrated signal. In fact, the normalized mean values in those 60 bins can themselves be used to get a reasonable estimate of impact velocity, without any information about the strength of the observed signal. The mean error is still large but as shown in figure 16, the errors are smaller for low velocities and hence fractional errors are reasonable for a large range of velocities. Whether this approach can be used to estimate the velocity of an impactor in space depends on the desired accuracy. For low speed impacts, an error of $6.56 \mathrm{~km} / \mathrm{s}$ might be too large but for large impact speeds, this method can be employed, provided the necessary calibration has been done at a groundbased hypervelocity impact test facility to determine the best parameters for the SVR model. 


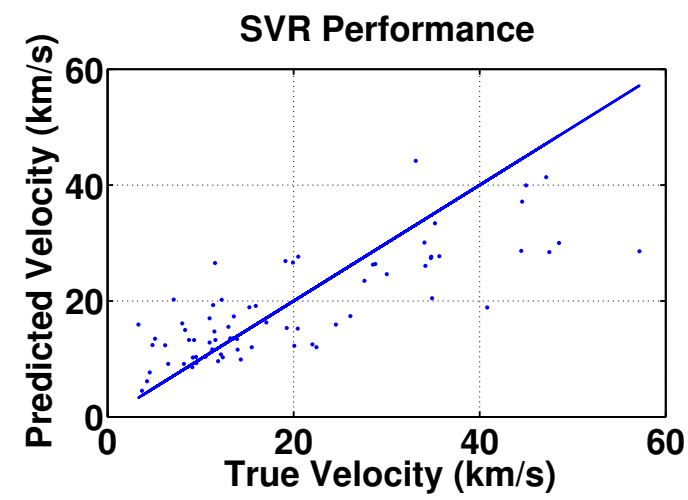

Figure 16: Predicted velocities vs true velocities for one of the cross-validation sets.

Since the masses of the projectiles are not independent of their velocities, an SVR approach in this case can also be used to estimate the mass of the impactor. Hence this technique cannot rule out the dependence of temporal characteristics on the mass of the impactor.

\section{Conclusion}

In this paper, we have presented an in-depth analysis of hypervelocity impact flash data obtained from seven different targets using a PMT at the Max Planck Institute for Nuclear Physics in Heidelberg, Germany. In the past, scaling laws that were developed to study the dependence of optical signal on the mass and velocity of the impactor either assumed the mass exponent to be 1 or calculated the mass exponent using a randomly chosen subset of the data. Using these non-robust methods, we were able to reproduce old results but a simultaneous estimate of mass and velocity exponents yielded velocity exponents less than 3 and mass exponents close to 0.5. In an effort to find a good estimator of impact velocity, we also demon- 
strated different approaches for studying the dependence of the temporal characteristics of the signal on the velocity of the impactor. We observed a significant negative correlation between the rise time of the integrated signal and velocity for velocities greater than $8 \mathrm{~km} / \mathrm{s}$. A correlation was also found between the rise time of the non-integrated signal and the velocity of the impactor. But in both these cases, the quality of the fit was poor and using them as estimators would yield very large errors in predicted velocities. The range of velocities over which the correlations exist and the type of relationship (affine vs power law) were also found to be contrary to what has been reported previously in literature.

Using a purely data-driven approach, we were able to estimate the velocity of the impactor with a mean estimation error of $6.56 \mathrm{~km} / \mathrm{s}$. Having estimated the velocity from the temporal characteristics of the optical signal, the mass of the impactor can be calculated using the scaling law for the strength of the optical signal. This allows us to use optical sensors to study the mass and velocity distributions of hypervelocity particles in space. These estimates can be further improved by combining measurements from optical and plasma sensors.

Few studies have examined the properties of the impact flash and hence more experiments are needed to fortify the results presented in this paper. For many of the targets, more impacts are needed to generate a large enough dataset to provide statistically significant estimates of various parameters. Simultaneously, numerical simulations are needed to arrive at estimates of signal duration for different impact parameters. If these simulations can associate impact parameters with other temporal characteristics of the ob- 
served signal, one can go and look for evidence of such correlations in the experimental data.

In the near future, more experiments will be executed at the Colorado dust accelerator facility where data will be acquired at multiple wavelengths to measure the evolution of the temperature of the target. Experiments will also be carried out at a light gas gun facility to determine if these results are consistent across several orders of magnitude variation in mass. These experiments will use an array of photodiodes since those are more suitable for eventually transitioning to a space-based experiment.

\section{Acknowledgements}

This study was sponsored by Los Alamos National Laboratory. The authors thank Ralf Srama, Sebastian Bugiel, Anna Mocker and other personnel of the Cosmic Dust Group at MPIK for their support during the experiments, and Justin Likar from Lockheed Martin for donating the spacecraft components that were used as impact targets. We also thank Pat Colestock from Los Alamos National Lab and Stan Green for supporting the experimental campaign and DOE (grant DE-SC0010390) for also supporting this project. During the course of this project, Ashish Goel was funded by the Stanford Graduate Fellowship program.

\section{References}

[1] Ceplecha, Z., Borovička, J., Elford, W., ReVelle, D., Hawkes, R., Porubčan, V., et al. Meteor phenomena and bodies. Space 
Science Reviews 1998;84(3-4):327-471. doi: \bibinfo\{doi\}\{10.1023/A: $1005069928850\}$.

[2] Pifko, S., Janches, D., Close, S., Sparks, J., Nakamura, T., Nesvorny, D.. The meteoroid input function and predictions of mid-latitude meteor observations by the mu radar. Icarus 2013;223(1):444-459.

[3] Grün, E., Zook, H.A., Fechtig, H., Giese, R.H.. Collisional balance of the meteoritic complex. Icarus 1985;62(2):244-272.

[4] Close, S., Colestock, P., Cox, L., Kelley, M., Lee, N.. Electromagnetic pulses generated by meteoroid impacts on spacecraft. Journal of Geophysical Research: Space Physics 2010;115(A12):- A12328.

[5] Fletcher, A., Close, S.. Model of plasma dynamics and electromagnetic pulses associated with hypervelocity particle impacts on satellites. In: General Assembly and Scientific Symposium, 2011 XXXth URSI. 2011, p. 1-4. ID: 1 .

[6] Lee, N., Close, S., Goel, A., Lauben, D., Linscott, I., Johnson, T., et al. Theory and experiments characterizing hypervelocity impact plasmas on biased spacecraft materials. Physics of Plasmas (1994-present) $2013 ; 20(3)$.

[7] Ju, Y., Zhang, Q., Zhang, D., Long, R., Chen, L., Huang, F., et al. Theoretical model for plasma expansion generated by hypervelocity impact. Physics of Plasmas (1994-present) 2014;21(9).

[8] Harano, T., Machida, Y., Fukushige, S., Koura, T., Hosoda, S., 
Cho, M., et al. Preliminary study on sustained arc due to plasma excited by hypervelocity impact of space debris on the solar array coupon. International Journal of Impact Engineering 2006;33(112):326-334.

[9] Close, S., Linscott, I., Lee, N., Johnson, T., Strauss, D., Goel, A., et al. Detection of electromagnetic pulses produced by hypervelocity micro particle impact plasmas. Physics of Plasmas (1994-present) $2013 ; 20(9)$.

[10] Enling, T., Qingming, Z., Jian, Z.. Preliminary study on magnetic induction intensity induced by plasma during hypervelocity impact. Chinese Journal of Aeronautics 2009;22(4):387-392.

[11] Tang, E.L., Zhang, Q.M., Wang, M., Xiang, S.H., Zhang, W., Yang, M.H., et al. Interference on logic chips due to plasma created by hypervelocity impact on 2024-t4 aluminum target. Chinese Journal of High Pressure Physics 2013;1:017.

[12] Grün, E., Fechtig, H., Kissel, J., Linkert, D., Maas, D., McDonnell, J., et al. The ulysses dust experiment. Astronomy and Astrophysics Supplement Series 1992;92:411-423.

[13] Thiessenhusen, K.U., Krüger, H., Spahn, F., Grün, E.. Dust grains around jupiterthe observations of the galileo dust detector. Icarus 2000;144(1):89-98.

[14] Eichhorn, G.. Measurements of the light flash produced by high velocity particle impact. Planetary and Space Science 1975;23(11):1519-1525. 
[15] Eichhorn, G.. Analysis of the hypervelocity impact process from impact flash measurements. Planetary and Space Science 1976;24(8):771-781.

[16] Burchell, M.J., Kay, L., Ratcliff, P.R.. Use of combined light flash and plasma measurements to study hypervelocity impact processes. Advances in Space Research 1996;17(12):141-145.

[17] Lawrence, R.J., Reinhart, W.D., Chhabildas, L.C., Thornhill, T.F.. Spectral measurements of hypervelocity impact flash. International Journal of Impact Engineering 2006;33(112):353-363.

[18] Thornhill, T.F., Reinhart, W.D., Chhabildas, L.C., Breiland, W.G., Alexander, C.S., Brown, J.L.. Characterization of prompt flash signatures using high-speed broadband diode detectors. International Journal of Impact Engineering 2008;35(12):1827-1835.

[19] Lawrence, R., Reinhart, W., Chhabildas, L., Thornhill, T., Kelly, D.. Hypervelocity impact flash for missile-defense kill assessment and engagement analysis: Experiments on z. Tech. Rep. SAND2005-3307; Sandia National Laboratories; Albuquerque, NM; 2005.

[20] Melosh, H.J., Artemjeva, N., Golub, A., Nemchinov, I., Shuvalov, V., Trubetskaya, I.. Remote visual detection of impacts on the lunar surface. In: Lunar and Planetary Science Conference; vol. 24. 1993, p. 975-976.

[21] Ortiz, J., Sada, P., Rubio, L.B., Aceituno, F., Aceituno, J., Gutierrez, P., et al. Optical detection of meteoroidal impacts on the moon. Nature 2000;405(6789):921-923. 
[22] Suggs, R.M., Cooke, W.J., Koehler, H.M., Suggs, R., Moser, D., Swift, W.R.. Lunar meteoroid impact observations and the flux of kilogram-sized meteoroids. In: Meteoroids Conference. 2011, p. 116124.

[23] Moser, D., Suggs, R., Swift, W., Suggs, R., Cooke, W., Diekmann, A., et al. Luminous efficiency of hypervelocity meteoroid impacts on the moon derived from the 2006 geminids, 2007 lyrids, and 2008 taurids. In: Meteoroids: The Smallest Solar System Bodies; vol. 1. 2011, p. 142.

[24] Serna, P.J.. Data report of hypervelocity micro-particle impact light flash data and mos impact detector output. Tech. Rep. ADA296741; Phillips Laboratory/WSCH; 3550 Aberdeen Ave, Kirtland AFB, NM),; 1995.

[25] Collette, A., Drake, K., Mocker, A., Sternovsky, Z., Munsat, T., Horanyi, M.. Time-resolved temperature measurements in hypervelocity dust impact. Planetary and Space Science 2013;89(0):58-62.

[26] Mocker, A., Bugiel, S., Auer, S., Baust, G., Drake, K., Fiege, K., et al. A 2MV Van de Graaff accelerator as a tool for planetary and impact physics research. Reviews of scientific instruments 2011;82:095111. doi: \bibinfo\{doi $\}\{10.1063 / 1.3637461\}$.

[27] Swift, W.R., Moser, D., Suggs, R.M., Cooke, W.. An exponential luminous efficiency model for hypervelocity impact into regolith. In: Meteoroids Conference. 2010, p. 125-141. 
[28] Eichhorn, G.. Primary velocity dependence of impact ejecta parameters. Planetary and Space Science 1978;26(5):469-471.

[29] Drucker, H., Burges, C.J., Kaufman, L., Smola, A., Vapnik, V.. Support vector regression machines. Advances in neural information processing systems 1997;9:155-161.

[30] Chang, C.C., Lin, C.J.. LIBSVM: A library for support vector machines. ACM Transactions on Intelligent Systems and Technology 2011;2:27:1-27:27. Software available at http://www.csie.ntu.edu. tw/ cjlin/libsvm. 